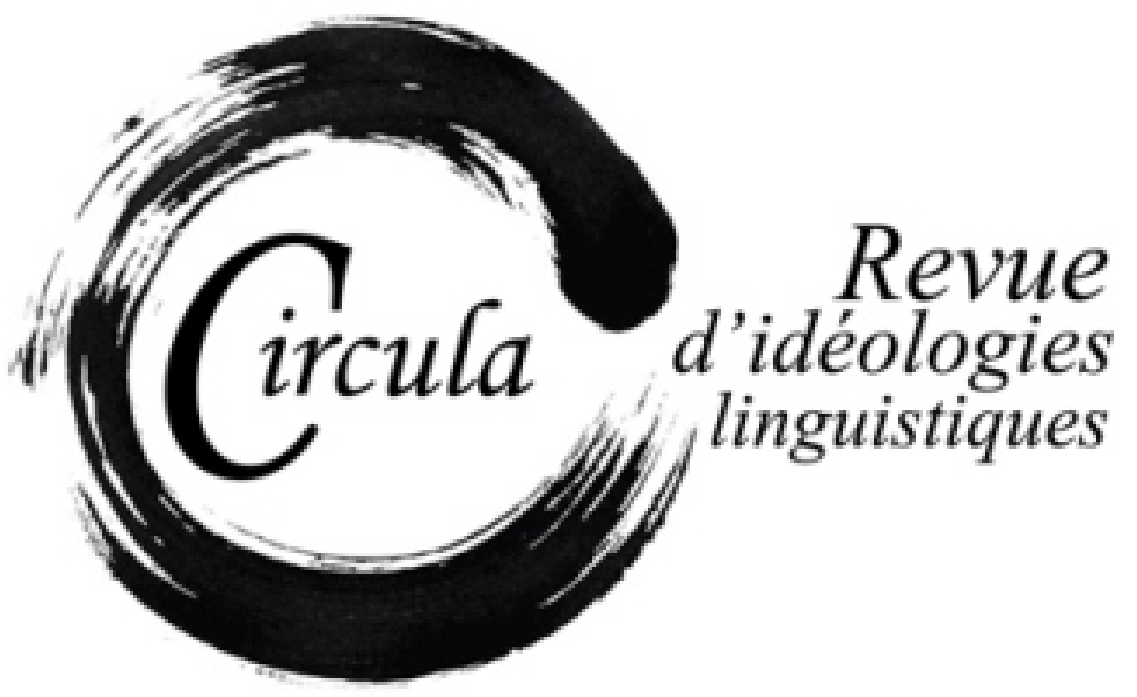

TITRE: LA LITTÉRATIE, UNE NOTION CHARGÉE IDÉOLOGIQUEMENT : LE TRAITEMENT DE L'ALPHABÉTISATION DANS LA PRESSE ACADIENNE

Auteure: LAurence ArRighi, Université de Moncton

Revue: CIRCULA, NUMÉRO 10

PAgES: $64-90$

ISSN: 2369-6761

URI: HTTP://HDL.HANDLE.NET/11143/16774

DOI: HTTPS://DOI.ORG/10.17118/11143/16774 


\title{
La littératie, une notion chargée idéologiquement : le traitement de l'alphabétisation dans la presse acadienne
}

\author{
Laurence Arrighi, Université de Moncton
} laurence.arrighi@umoncton.ca

[...] la langue sert de terrain de sélection mystifiable, puisqu'on avance la proposition qu'elle est accessible, tout en maintenant des mécanismes inégalitaires de définition de la compétence, mécanismes désavantageux pour tous ceux et toutes celles qui se font évaluer, et n'évaluent pas. (Heller, 2007 : 44)

Résumé : Une familiarité avec les discours sur la langue dans la presse acadienne m’a conduit à porter une attention particulière à un discours récurrent sur la question du niveau de littératie des francophones du Nouveau-Brunswick et des besoins en alphabétisation des membres de cette communauté. Par une analyse critique de discours, et en pensant avant tout la littératie comme une pratique sociale, je mets en lumière la charge idéologique de cette notion et de tout discours portant sur les questions d'alphabétisation. En lien avec la tendance grandissante dans nos sociétés à évaluer les pratiques linguistiques comme des compétences mesurables, ainsi que dans un contexte plus large de dénigrement permanent des pratiques du groupe étudié, je mets de l'avant la manière dont la notion de littératie et la question de l'alphabétisation sont investies discursivement, soit par un discours de l'emphase soit par un traitement intimiste, tous deux largement orientés idéologiquement. In fine, je cherche à déceler quelle(s) grande(s) idéologie(s) servent les discours étudiés.

Mots-clés : langue française en Acadie ; littératie ; alphabétisation ; idéologies linguistiques

Summary: Familiarity with language discourses in the Acadian Press led me to pay special attention to a recurrent discussion on the issue of the literacy level of Francophones in New Brunswick and the literacy needs of members of this community. Using critical discourse analysis, and analyzing literacy primarily as a social practice, I highlight the ideological nature of this position, and indeed of all discourses on literacy. After highlighting the growing tendencies of our societies toward the evaluation of linguistic practices as measurable skills, and the denigration of the practices of the group studied, I underline the extent to which the very notion of literacy is invested discursively and oriented ideologically. In short, I attempt to discover which ideology or ideologies the discourse studied serves.

Keywords: French language in Acadia; speeches on literacy; linguistic ideologies 


\section{Introduction ${ }^{1}$}

L'objectif de cette contribution est de présenter le traitement médiatique de la question de l'alphabétisation et, plus largement, du thème du niveau de littératie des francophones en Acadie. Les outils de la Critical discourse analysis (Fairclough, 1995) seront utilisés afin de comprendre un discours en général très consensuel sur les besoins en alphabétisation dus au niveau de littératie tenu pour piètre des francophones du Nouveau-Brunswick. Mon approche sera également nourrie par les théories de l'argumentation ainsi que par des travaux produits en sociologie de l'éducation et dans d'autres sciences sociales.

Mon travail sur les discours traitant de la question de l'alphabétisation en Acadie m'a amenée à comprendre que, dans les espaces discursifs (presse, discours gouvernemental, discours d'associations, etc.) de la francophonie acadienne, les concepts somme toute savants d'alphabétisation et de littératie sont utilisés sans plus être définis. Cet article interroge ces notions voire les remet partiellement en cause, celles-ci s'étant transformées, au fil des années, en un enjeu idéologique au profit duquel on est appelé à militer. A minima, il convient de ne pas oublier, comme le rappellent Collins et Blot dans Literacy and literacies : text, power and identity (2003), que les pratiques de littératie sont, comme toutes pratiques et performances socioculturelles, interdépendantes des ordres sociaux et des rapports de pouvoir particuliers où elles apparaissent².

En l'occurrence, la communauté dont il est question est celle des Acadiens et Acadiennes du Nouveau-Brunswick. Cette communauté minoritaire de l'est du Canada forme environ un tiers de la population face aux Anglophones; elle est périphérique au sein de la francophonie canadienne et qui plus est mondiale.

Certains motifs discursifs dans le traitement médiatique de la question de l'alphabétisation en Acadie analysés ici peuvent relever plus spécifiquement de la situation acadienne - c'est souvent ainsi que cela est posé - je montrerai toutefois certaines convergences avec d'autres situations. Je propose surtout que ce discours est à comprendre plus largement dans deux directions. Premièrement, il s'inscrit ici dans un discours plus vaste et récurrent de dénigrement et d'autodénigrement des pratiques linguistiques acadiennes (Boudreau, 2009, 2012, 2016 ; Arrighi et Violette, 2013 ; Arrighi et Urbain 2016/2017 et 2019). Deuxièmement, il se comprend comme épiphénomène d'une tendance grandissante dans nos sociétés à évaluer les pratiques linguistiques comme des compétences mesurables. Ce faisant, mon propos s'inscrit dans le cadre plus général d'une réflexion en cours sur les questions de langue qui consiste à interroger les conséquences de cette propension

1. Je tiens à exprimer ma reconnaissance à Émilie Urbain (Carleton University) pour ses conseils lors de la rédaction de cet article et à Naorën Vernouillet (Université de Moncton) pour l'aide précieuse tout au long de la collecte des données. Merci aussi aux deux évaluateurs/évaluatrices pour la révision minutieuse et les suggestions fort pertinentes.

2. Dans le sillage des New Literacy Studies (Street, 1996 et 2005) ma démarche appréhende la littératie « focusing not so much on literacy as a "technology of the mind" [...] or as a set of skills, but rather on what it means to think of literacy as a social practice » (Street, $2006: 1$ ). 
croissante à l'évaluation tout azimut des usages de la langue, mais in fine des individus. Ces études conduites surtout dans le sillage de la sociolinguistique critique montrent comment ces discours participent de processus de régulation sociale, hiérarchisent les locuteurs, sont naturalisés et posés comme un « allant de soi » sans être interrogés (voir Canut et Duchêne, 2011; Flubacher et Del Percio, 2017 ; Hambye et Romainville, 2013 ; et, spécifiquement pour le groupe acadien, Boudreau, 2016 ; McLaughlin, 2014). La présente contribution s'inscrit également dans ce courant, dans la mesure où je propose d'analyser ces discours en interrogeant les motivations des acteurs sociaux qui les tiennent, le cadre idéologique à partir duquel ils parlent, leurs enjeux et conséquences.

Dans cette contribution, je m'intéresse, dans un premier temps, à la façon générale dont le problème du niveau de littératie déficient des francophones en Acadie est construit discursivement. Dans un second temps, je regarde comment l'analphabète est représenté quand on s'attarde au cas particulier. En l'occurrence alors, c'est surtout l'ex-analphabète, devenu modèle de réussite et de résilience, qui est présenté. De fait, selon un mouvement déjà mis au jour dans d'autres travaux portant sur les discours sur la qualité de la langue (Arrighi et Violette, 2013 ; Arrighi et Urbain, 2016/2017), et plus largement les questions linguistiques, on passe de l'(auto-)dénigrement à l'éloge de ceux qui ne ménagent pas leurs efforts pour sortir de leur situation.

Avant cela, je propose, en guise de succincte contextualisation de la situation de la communauté, un bref aperçu des topos qui la traversent quand on en vient à traiter de la langue des Acadiens et des Acadiennes. Dans cette section, je présente aussi la presse francophone en Acadie. Nous verrons qu'elle est réduite à peu de choses, ce qui ne favorise pas la diversité d'opinion et peut certainement participer à expliquer la grande cohérence, pour une époque donnée, dans les propos tenus sur le sujet qui nous occupe. Enfin, dans cette section introductive, je m'arrête sur le cheminement des termes étudiés.

\section{Des pratiques linguistiques, de la presse francophone et de l'ap- parition du thème en Acadie}

De façon très générale, les Acadiens et les Acadiennes possèdent un répertoire langagier pluriel, dont un français qui peut être mêlé d'anglais et marqué de particularismes divers (Neumann-Holzschuh et Mitko, 2018). Cela est loin d'être perçu positivement et, à partir notamment de valeurs associées aux idéologies du monolinguisme et du standard, un certain discours public pointe des défauts de leur langue allant jusqu'à interroger leur qualification comme francophones (Arrighi et Boudreau, 2013, 2016 ; Boudreau, 2016). Au sein d'un concert de propos - souvent assez désobligeants - évaluant leurs pratiques linguistiques, le sujet de leur faible niveau d'alphabétisation et d'une littératie défaillante est récurrent. Le traitement discursif de ce thème présente une étroite parenté avec d'autres discours sur la forme, maitrise et qualité de la langue de ce groupe (Arrighi et Boudreau, 2013 ; Arrighi et Violette, 2013). Ce sont précisément mes analyses antérieures des discours publics sur la langue qui m’ont amenée à choisir d'analyser ce thème. Ce qui a également attiré mon attention, c'est la 
singulière unanimité dans le diagnostic : le niveau de littératie est au plus bas, les besoins en alphabétisation sont immenses. On relève aussi une certaine uniformité de ton et de formulation. Ce sont souvent les mêmes antiennes qui reviennent et elles sont fort proches de celles relevées dans d'autres espaces quand il est question d'alphabétisation (ainsi, en France, le travail de Lahire, 1999, 1992) ; or, comme l'ont déjà noté des chercheurs et chercheuses travaillant sur ce sujet sur d'autres terrains, entre la réalité des inégalités d'accès à l'écrit, bien réelles, et les discours qui en traitent, le rapport n'est pas toujours évident (Lahire, 1999).

Autant dans la situation commentée par Lahire que dans les journaux formant mon corpus, on assène des images choc : « $60 \%$ des francophones de la province auraient du mal à comprendre tous les articles qui se trouvent dans le journal d'aujourd'hui » (AN, 22 septembre 2016³). L'objet mis en discours a de quoi choquer et c'est bien l'objectif des discoureurs. Dans de nombreux textes, les auteurs montent au créneau pour dénoncer le « fléau » (AN, 22 septembre 2016) de l'illettrisme en Acadie, mal auquel le " public doit être sensibilisé » (AN, 25 juin 2011) et contre lequel il faut « partir en croisade » (AN, 12 mai 2000). L'on a affaire à un discours peu nuancé et assez homogène où l'espace du dicible sur le sujet est en quelque sorte prédéfini, comme si l'on avait affaire à une formation discursive qui « détermine ce qui peut et doit être dit» (Haroche, Henry et Pêcheux, 1971 : 102). Comme tout discours, contraint par ses conditions de production (Foucault, 1971), le discours public sur l'alphabétisation semble ce faisant «menacé d'exclusion s'il déborde du cadre permis et interprété quant au contenu, à la discipline, à la validité de son énonciation » (Achard, 1986 : 16).

Précisons qu'il ne s'agit avec ce travail ni de poser un nouveau diagnostic qui invaliderait celui que je viens de souligner, ni de balayer du revers de la main les efforts combinés des organismes en charge de l'alphabétisation et des bénéficiaires de ces services. Toutefois, il est sans doute important aujourd'hui de prendre du recul vis-à-vis de procédures évaluatives peu transparentes et, quoi qu'il en soit, mal comprises. Il convient de se demander qui mesure qui, quoi, comment et pourquoi pour en arriver aux chiffres cités et avec quelles conséquences ? II convient aussi d'interroger les discours qui valident ces évaluations, des propos trop consensuels et des démarches, certes généreuses, qui consistent à promouvoir une meilleure littératie, mais sans que cette notion soit plus avant définie. En fait, définie de différentes façons par divers courants de la recherche ${ }^{4}$, la littératie (et l'alphabétisation) a, sans conteste, besoin d'être critiquée scientifiquement pour être (ré-)affinée et surtout pour ne pas s'instituer comme doxa sociétale et pédagogique dont les protagonistes principaux, agents, agentes et bénéficiaires des services d'alphabétisation, ne comprendraient ni les tenants ni les aboutissants; ni les lecteurs et lectrices de la presse qui en traite d'ailleurs. On peut aussi interroger les positionnements parfois trop angéliques de ces discours et démarches. Sur un autre terrain

3. Dans le corps du texte les références au corpus se lisent comme suit : initiales du titre de presse et date de parution de l'article cité. En bibliographie, chaque référence est détaillée.

4. Je précise ici que, travaillant à partir d'un corpus journalistique, je n'entrerai pas dans un examen critique ni même une présentation de l'usage de ces termes dans le monde académique ou au sein des institutions. Très certainement productive, la confrontation des usages entre différents milieux, et au sein même d'un seul milieu, n'est pas du ressort de cet article. 
où règne un consensus qui frise la doctrine : la promotion institutionnelle de l'éducation plurilingue en Europe, des chercheurs et chercheuses qui par ailleurs sont parfois fortement engagés dans cette voie ne se privent pas de souligner le caractère convenu, angélique ou simplificateur de discours sur le thème. Ce faisant, ils enrichissent notre compréhension des défis qui se posent à ceux et celles qui veulent agir. Ce type de réflexion, en particulier celle de Clerc et Rispail (2008), a enrichi la mienne.

Je propose, comme l'indique mon titre, de considérer la littératie, telle que construite discursivement pour le grand public, comme une notion chargée idéologiquement. À la fin de ma contribution, je reviendrai sur cette proposition en m'interrogeant notamment sur l'idéologie ou les idéologies qui sauraient servir par des discours sur le niveau de littératie et les besoins en alphabétisation de tel ou tel groupe.

Ce n'est donc pas le concept savant de littératie 5 , ni les pratiques d'alphabétisation que j'interroge mais la façon dont il est souvent question de ces sujets auprès du grand public. Je vise à en montrer le caractère idéologiquement situé en lien avec des représentations plus générales sur les pratiques linguistiques en Acadie. On ne peut faire abstraction, en effet, du fait que l'on est face à un discours qui, comme toute prise de parole, ne s'inscrit pas dans un vacuum mais s'insère dans un paysage discursif particulier. En l'occurrence, la communauté étudiée est particulièrement en butte à des représentations négatives quand on en vient aux pratiques linguistiques de ses membres.

Bien des travaux ont pu montrer le rôle prépondérant de la presse dans l'élaboration et la diffusion d'idéologies à caractère linguistique (Johnson et Esslin, 2007 ; Johnson et Milani, 2010). De fait, en Acadie, la presse francophone est un véhicule de premier plan pour proposer et débattre de questions linguistiques. Plusieurs travaux avant le mien ont souligné et analysé le rôle patent des journaux et autres médias acadiens en la matière, dont Boudreau $(2009,2011,2012)$ et Boudreau et Urbain (2013).

Voici comment ces dernières justifient et expliquent le recours à la presse et en l'occurrence à la presse acadienne :

Le discours de la presse en milieu minoritaire est un objet d'étude d'autant plus pertinent que les journaux et les périodiques ont souvent été, pour les groupes historiquement dominés ou opprimés, une voie d'accès privilégiée au discours public [...]. Comme l'ont illustré certains travaux en Critical Discourse Analysis (CDA) ou en étude des communautés minoritaires, le fait même d'avoir accès à une prise de parole publique, et d'autant plus écrite ou imprimée - que ce soit comme producteurs ou objets des discours - illustre souvent des rapports de force entre différents groupes d'acteurs sociaux. Ces inégalités dans l'accès aux ressources symbo-

5. La littératie est, à l'instar de tout concept, sujette à une définition mouvante. Il n'en demeure pas moins qu'elle est parfois saisie de façon rigide et univoque. En fait, ou on ne la définit pas (on la prend comme un allant de soi) ou on la définit trop. Pareillement, vu que l'alphabétisation est empreinte d'idéologies, linguistiques et autres (Barton, Hamilton et Ivanić, 2000 ; Street, 1995), elle ne se réduit pas à une opération technique visant l'acquisition de compétences linguistiques. 
liques que constitue le discours de presse ont de nombreuses conséquences sur le plan des dynamiques sociolinguistiques et posent toutes une série de questions : qui s'exprime et qui peut s'exprimer (qui a le capital symbolique pour le faire) ? Quels sont les intérêts des acteurs qui prennent la parole? Quelles sont les stratégies adoptées, en visant quel public et dans quel but? (Boudreau et Urbain, 2013 : 25)

J'adopte dans ma recherche une perspective historique dans le but de suivre le cheminement du thème qui me préoccupe. Pour ce faire, j'opère un dépouillement exhaustif de trois journaux acadiens qui se sont succédé (où ont parfois coexisté) de 1867 à nos jours. Il s'agit de l'Acadie nouvelle, quotidien fondé en 1984 et toujours en activité ; de l'Évangéline qui publia de 1887 à 1982 ; du Moniteur acadien, fondé l'année même de la Confédération, il publiera des numéros de façon pas toujours quotidienne jusqu'en 1926. Dans la mesure où désormais plusieurs travaux ont porté sur ces trois organes de presse, j'invite le lecteur à consulter les titres opportuns ${ }^{6}$. On retiendra une offre de titre limitée qui ne permet peu d'études contrastives mais qui témoigne du rôle central de chacun de ces organes de presse pour la communauté.

Mon exploration de ces journaux se veut exhaustive. L'ensemble des numéros des journaux visés est dépouillé électroniquement ou manuellement, selon les journaux et les périodes visées. J'ai recherché tout écrit contenant les mots alphabétisation et dérivés, littératie (y compris sous la graphie littéracie), illettré et ses variantes et de façon plus aléatoire tout article consacré à la pratique de la lecture en Acadie ${ }^{7}$. Cette perspective historique me permet d'emblée de dire que le traitement de la question de l'alphabétisation a changé. Il y a eu en effet des tournants dans le traitement du thème, dont un majeur : quelque part au milieu des années 1990, l'alphabétisation a été constituée comme un problème social actuel et local. De fait pendant longtemps, l'analphabétisme c'était le problème des autres : un simple sujet mentionné à la faveur d'une actualité plaisante, ainsi dans l'Évangéline du 22 juillet 1977 on trouve une nouvelle quelque peu sensationnaliste : des étudiants de l'Université de Calgary ne sauraient pas lire, le 23 septembre de la même année c'est le cas similaire d'un diplômé de Seattle qui est mentionné. Le sujet est traité de façon bien moins anecdotique quand il s'agit de «l'analphabétisme dans le monde », à savoir dans les pays pauvres, pays pauvres qui sont alors à soutenir dans un mouvement empreint d'un colonialisme encore récent (plusieurs lettres ouvertes dans L'Évangéline vont dans ce sens, voir par exemple l'édition du 30 septembre 1971). Dans L'Évangéline daté du 16 mai 1962, c'est la situation préoccupante en matière d'analphabétisme en Amérique du sud, Asie et Afrique qui est portée à l'attention des lecteurs et lectrices. On souligne alors que cette réalité est inconnue dans les « pays riches, dont le Canada ». On met enfin de l'avant

6. Sur L'Acadie Nouvelle, voir Eddie (2011), sur L'Évangéline, voir Beaulieu (1997) et Eddie (2015). Voir aussi Beaulieu (1993) et Watine (1992 et 1993). Ces contributions éclairent notamment sur le lectorat et la politique éditoriale de ces organes de presse.

7. Le corpus récolté jusqu'ici (plusieurs années restant à reconsidérer) comprend 763 documents de presse (articles, éditos, lettres ouvertes, annonces publicitaires). 
le rôle capital que peut jouer l'Église catholique pour remédier à cette situation. Dans L'Évangéline du 24 octobre 1978, c'est le cas de l'Égypte qui est mis de l'avant, etc.

Bien entendu, il faut garder en tête qu'avant la fin du dernier quart du XXe siècle la notion de littératie (a fortiori le terme lui-même en français) n'était pas vraiment en circulation. De plus, à toute époque, l'on pouvait parler du niveau d'alphabétisation sans utiliser le mot. Ceci étant, les parties du corpus qui ont dû être dépouillées manuellement (les numéros de L'Évangéline entre 1959 et 1982 et ceux de L'Acadie Nouvelle de 1998 à 1984) me permettent de poser qu'il y a un changement observable.

Une transition discursive a donc lieu dans la presse acadienne au début des années $1990^{8}$. Selon la littérature sur le sujet pour la France, l'analphabétisme comme problème national pointe dans les médias et plus largement dans le discours public à partir du milieu des années 1980 (voir les études de Lahire 1992, 1999 ; voir aussi Espérandieu et Vogler, 2000). Si, en Acadie, il semble arriver un peu plus tard, le sujet s'impose avec la même rhétorique destinée à en démontrer la gravité. À partir de la moitié des années 1990, l'alphabétisation fait l'objet comme problème social d'une construction discursive. On peut ainsi établir nombre de parallèles avec le travail de Gusfield (1981) sur la construction sociale du problème de l'alcool au volant. Dans The Culture of Public Problems (1981), le sociologue cherche en effet à comprendre pour quelles raisons et sous quelles conditions la conduite automobile sous l'emprise de l'alcool est devenue une préoccupation sociétale. Il observe les conditions d'émergence de cette question, ensuite la constitution, la stabilisation et l'institutionnalisation.

À partir de mes données, je propose que l'on regarde cette période où l'alphabétisation est devenue un problème social ${ }^{9}$. Nous verrons alors que ce sujet est traité le plus souvent de deux façons presque opposées. On en propose une approche que je qualifierai de discours d'alarme où la gravité de la situation est posée à coup d'experts, de données quantitatives via la statistique et d'études internationales, nationales ou régionales convoquées pour poser la gravité de la situation. Le propos en est souvent un de condamnation et d'appel à réagir. Ce sont alors le plus souvent les mêmes ingrédients que dans d'autres discours sur la qualité de la langue en Acadie que l'on retrouve (appel à l'effort individuel, incrimination du bilinguisme et plus particulièrement du mélange de codes, blâme individuel ou collectif pour un manque de fierté linguistique, etc...). De façon contrastante, on trouve également un traitement que je qualifierai de discours d'espoir essentiellement qualitatif,

8. Jusqu'à un certain point, il est possible de relier l'apparition du thème à l'universalisation de la mesure de l'alphabétisation par les instances internationales type OCDE.

Sur un autre plan notons que le premier organisme en charge de l'alphabétisation au Nouveau-Brunswick (APA, Association provinciale d'alphabétisation) voit le jour en 1988 (voir L'Acadie nouvelle du 3 aout de cette année-là).

9. Mon objectif avec cet article n'est pas de proposer une explication d'ordre social et politique au cheminement du thème. J'adhère à une vision critique des politiques d'alphabétisation que je peux résumer ainsi : les transformations globales qui affectent, au moins depuis le dernier quart du XXe siècle, tous les secteurs professionnels induisent pour quasiment tous les postes de travail un usage professionnel de la langue, sous forme écrite et/ou orale, cette « part langagière du travail » (Boutet, 2001, 2007) appelle les tats à capitaliser sur les compétences linguistiques de leurs citoyens. Ceci étant, je conclurai ma contribution en présentant ce que je considère comme une clé de compréhension de la prégnance du thème en Acadie. 
très personnalisé, qui passe en particulier par l'exercice du portrait ou de l'autoportrait. L'espoir se donne alors à voir par le récit de parcours individuels particulièrement résilients. Ce sont ces deux façons d'aborder la question que je propose de regarder maintenant ${ }^{10}$.

\section{Le traitement alarmiste}

Dans le corpus de presse sur lequel je travaille, ceux qui traitent du sujet du niveau de littératie en Acadie en font très souvent un « sujet chaud ». Ils dressent un tableau alarmant qui vise à interpeller, émouvoir, choquer. On a affaire à un « cadrage de crise » tel que le décrit Cameron (2007) lorsqu'un sujet n'a pas directement les caractéristiques qui en font une «news value ». Cette technique qui marque bien des discours sur la langue dans les médias est employée pour faire d'un sujet a priori peu médiatique, un sujet mainstream et digne d'intérêt. Chez Cameron, il est question du thème des langues en danger et ce qu'elle décrit est très nettement applicable à mon sujet. La description est un peu longue mais mérite d'être citée :

During the past ten years, the plight of endangered languages has attracted increasing interest from the mainstream media. [...] Since the representation of any issue for a mass audience has implications for the way it is understood, it is of interest to inquire how - that is, through what kind of representation - language endangerment has been able to move into the mainstream. A useful parallel here is with climate change [...]. One thing the two issues have in common is that they do not fit the prototype for news stories [...]. To make such issues newsworthy, it is necessary to inject drama and urgency by framing them as grave crises which we ignore at our peril. But while « crisis » framing may give an issue greater mainstream visibility, it can also lead to unbalanced and misleading coverage [...] In an effort to fit the facts to the frame, statements about to extend of the problem may be reproduced shorn of the caveats their expert sources were careful to include ; disagreement among experts may be glossed over in formulations like « many scientists believe... »; prominence may be given to the most extreme and alarmist expert predictions, or even to predictions no expert has made, but which journalists have extrapolated from their inexpert reading of the evidence. (Cameron, 2007 : 268-269)

Nous allons voir que nous retrouvons des éléments similaires dans le traitement de notre sujet. Premier ingrédient qui permet de poser un diagnostic en apparence alarmant: l'utilisation de termes qui ne sont pas toujours transparents. Il est temps de faire le point sur les mots du débat : littératie et alphabétisation. Plusieurs sources montrent que l'usage du terme littératie explose après les années 1980. Malgré sa popularité, le terme reste encore un mot savant dont il n'est pas toujours aisé de dé-

10. Dans une contribution issue d'une recherche sur le milieu de l'alphabétisation en Ontario français, Budach (2003) identifie trois types de discours sur l'alphabétisation : un discours de l'alphabétisation populaire, un discours de l'alphabétisation culturelle et un discours de l'alphabétisation bureaucratique. Elle indique que chacun « exerce un certain pouvoir de domination à un moment donné de l'histoire et des débats sur l'alphabétisation aux adultes en Ontario. » (2003: 337). Il se peut qu'une telle répartition se retrouve dans mes données. Cette piste est certainement à creuser. 
finir les contours. La littératie a été qualifiée par l'OCDE de comportement, d'aptitude ou de capacité des adultes à utiliser et à comprendre l'écrit au quotidien. Elle est devenue ensuite une compétence clé en traitement de l'information. Cette notion « englobe une variété de compétences, depuis le décodage de mots et de phrases jusqu'à la compréhension, l'interprétation et l'évaluation de textes complexes» (OCDE, 2013:64) ${ }^{11}$. On comprend donc que littératie n'est pas équivalent d'alphabétisme et qu'un déficit de littératie ne fait pas de l'individu un analphabète. En fait l'individu en déficit de littératie serait un illettré mais le néologisme lettrisme (et ses dérivés), favorisé contre l'emprunt, n'a pas accroché au Canada français ${ }^{12}$. Les choses se compliquent dans la mesure où ce qui est proposé pour lutter contre un déficit de littératie, c'est de l'alphabétisation (et non de la *littérarisation). Ainsi, on en arrive souvent dans le discours à un certain mélange des genres où la non-équivalence entre alphabétisme et littératie n'est plus claire pour le lectorat. Est-elle claire pour celui qui mobilise ces termes? En fait, cette question n'est pas abordée minutieusement et techniquement mais souvent à grands traits. L'efficacité du propos compte sur d'autres ingrédients. D'abord, pour affermir son dire, le discoureur (auteur de l'article, expert cité dans l'article) construit son éthos. II se présente comme un citoyen éminemment concerné, à la conscience civique et communautaire doublée en général d'une expertise de premier plan parce que peu ou prou spécialiste de la langue (enseignant, écrivain, ...). On livre donc un discours de l'auctoritas. Nous avons aussi affaire à un discours de l'emphase : le problème est important quantitativement (plus de $60 \%$ d'inaptes à lire L'Acadie Nouvelle), mais il y a aussi sa dimension qualitative, ses conséquences: l'analphabète, le peu-lettré est dépourvu de toute capacité expressive, sans ressources linguistiques, enfermé dans l'inculture de l'oral, privé de toute prise de pouvoir sur sa vie, ce qui le conduit nécessairement vers les crises familiales à répétition (il est un parent dysfonctionnel, une famille monoparentale), la malnutrition (il est ou va devenir obèse, diabétique ..., il coûte cher au système de santé), le sous-emploi (inutile dans une société du savoir), le désintérêt citoyen (il ne vote pas), etc. Avant tout, on assène des chiffres écrasants :

Autre statistique alarmante, M. Jeune souligne «qu'en 2003, selon la dernière recherche menée par l'Enquête internationale sur l'alphabétisation et les compétences des adultes (EIACA), au N.-B., 66 \% des Acadiens et francophones se situaient dans les deux niveaux d'alphabétisme les plus bas! Ceci confirme l'importance d'une Journée internationale de l'alphabétisation afin d'informer la population qu'encore en 2011, la situation persiste, et même s'aggrave dans certains niveaux d'âge. » (AN, 8 septembre 2011)

11. Si la littératie fait l'objet de définitions institutionnelles changeantes c'est précisément parce qu'elle est un objet de lutte idéologique. Je ne débats pas ici de ses acceptions dans le discours institutionnel, ni dans le discours savant. Je présente toutefois a minina, quelques éléments définitoires, voir notamment les notes 2 et 4 , voir aussi ci-dessus).

12. Voir la fiche terminologie qui est consacrée à ce terme par l'Office québécois de la langue française, http://www. granddictionnaire.com/ficheOqlf.aspx?ld_Fiche=8363201 (fiche consultée le 23 avril 2019). 
une majorité de Néo-Brunswickois se situe sous la moyenne nationale en matière d'alphabétisme. La situation est pire chez les francophones de la province puisque $66 \%$ d'entre eux ont un niveau de lecture inférieur à 3 , niveau considéré comme étant nécessaire afin de fonctionner dans une société du savoir (AN, 8 septembre 2008)

Les chiffres sont éloquents. Au Nouveau-Brunswick, les deux tiers des adultes n’ont pas le niveau de littératie nécessaire pour fonctionner dans un milieu de travail. À l'école, de 20 à $30 \%$ des élèves ont de la difficulté à lire au niveau souhaité pour leur âge. (AN, 28 janvier 2014)

Selon la formule les chiffres parlent d'eux-mêmes, de nombreux articles sont émaillés de chiffres. Ceux-ci sont, comme toutes statistiques, fondés sur des méthodes d'échantillonnage. Ils sont pourtant donnés pour la réalité alors que ce qu'ils sont vraiment ce sont des estimations, estimations pas absolument représentatives dans la mesure où une partie ne peut jamais valoir pour le tout. Ces résultats, en passant du champ de l'expertise au champ médiatique, se simplifient encore. Ce faisant, ils captent l'attention. L'emphase se retrouve aussi sous la forme d'énumération des conséquences et des implications de ce problème : «l'analphabétisme cache souvent des problèmes plus profonds, allant des troubles d'apprentissage aux difficultés socioéconomiques qui constituent souvent le quotidien de cette population en quête d'un avenir meilleur » (AN, 9 septembre 2011). L'auteur peut proposer ensuite des solutions simples à des problèmes complexes. Si le taux de bonne littératie remonte, tout peut être réglé :

la province doit investir en littératie et en alphabétisation si elle veut voir des citoyens en bonne santé.

«Pour que la province devienne autosuffisante, il faut faire de l'alphabétisation un dossier prioritaire afin d'avoir des personnes en santé et une province en santé à tous les points de vue », a dit M. Doiron [...]

[selon la FANB] « l'alphabétisation est un déterminant de la santé globale » d'une population. Pour appuyer ses propos, l'organisme souligne que de nombreuses études ont montré que les gens aux prises avec des difficultés de lecture ont habituellement plus de difficulté à trouver un emploi bien rémunéré.

Les difficultés de lecture entraînent toutefois aussi des problèmes de santé. L'organisme explique que les gens avec un faible taux de littéracie éprouvent souvent des problèmes à repérer et à comprendre de l'information médicale. Ces difficultés peuvent entraîner l'incapacité de comprendre " une posologie ou encore des conseils médicaux ». À cet effet, le Conseil canadien sur l'apprentissage a établi un lien entre certaines maladies chroniques et la littératie en santé.

La FANB souligne que de telles difficultés de compréhension ont des répercussions sur les coûts du système de santé. [...] 
Ainsi, une étude américaine a montré que le coût nécessaire pour soigner un patient avec un bon taux de lecture est en moyenne deux fois moins élevé que le coût nécessaire pour soigner un patient qui éprouve des difficultés de lecture. (AN, 8 septembre 2008)

Ce qui caractérise ce discours, ce sont des inductions, généralisations, pseudo-évidences appuyées sur quelques chiffres ainsi que sur de «nombreuses études » souvent « américaines » dont on extrait quelques faits «choc ». On assène de grandes phrases, prêtées à des autorités, des écrivains souvent, venus d'ailleurs, ce qui assure leur autorité. Un journaliste nous rappelle ainsi que : «Prendre plaisir à la lecture vaccine contre l'échec à l'école et dans la vie. Un enfant n'aimant pas la lecture est en danger», avait exprimé Alexandre Jardin [lors de son passage à Moncton] » (AN, 28 octobre 2014). En fait, les mêmes ingrédients reviennent sans cesse : poids des chiffres, parole rapportée d'experts pas toujours nommés, mention d’organismes aux noms éloquents, renvoi à des études qui ont toute l'apparence du plus grand sérieux et dont on extrait des citations tronquées jamais référencées : « une étude, une récente étude, une étude américaine, de nombreuses études... » qui ont toutes « montré, démontré, prouvé ». On note aussi une multiplication des termes : analphabétisme, niveaux 2 ou 3 d'alphabétisation, analphabétisme fonctionnel, défaut de littératie, ... Les appellations ne manquent pas, mais ce qu'elles recouvrent n'est pas toujours clair. À la lecture des articles, il ressort un flou terminologique qui explique l'alternance souvent aléatoire de ces diverses appellations alors qu'il n'est pas aisé de cerner ce que les termes veulent dire pour leurs usagers et comment ils caractérisent ceux qui en sont affublés. Enfin, quand on en vient à proposer des explications des faits que l'on vient de poser c'est, comme souvent quand il est question de langue et de qualité de la langue en Acadie, sur le compte de la situation minoritaire que la faute retombe. À partir de l'idéologie très ancrée du monolinguisme (Boudreau, 2009), la présence de l'anglais dans la vie des Acadiens et des Acadiennes serait en cause. Ainsi, dans une lettre ouverte, le chiac est pointé :

puisque Acadieman, qui parle le chiac, est proposé à l'humanité comme " héros acadien », il conviendrait de se demander : «Quelle philosophie de vie sociale et culturelle, quelles valeurs humaines, civiques et spirituelles sont véhiculées par ce personnage ? » [...]

Faire la promotion du chiac dans un contexte scolaire, sans comprendre suffisamment, au préalable, la place qui lui revint en Acadie et l'utilisation que l'on doive en faire dans l'ensemble de la Francophonie risquerait, je le crains, d'enfreindre les efforts déployés vers la maîtrise du français standard, au niveau de l'alphabétisation des adultes et dans la lutte contre l'assimilation. (AN, 19 février 2009)

Au milieu de la kyrielle des maux linguistiques qui guettent les francophones en Acadie, il y a le mélange de langues, la difficile maitrise du français standard, le bas niveau d'alphabétisation qui n'a d'équivalent que le haut niveau d'assimilation. On fait comme si le diagnostic était imparable et le propos n'est plus interrogé, on parle sur le mode du constat : « le milieu minoritaire dans lequel on vit [est] très en arrière en termes d'alphabétisation et de littératie » (AN, 28 octobre 2014). Ce discours d'évidence fait partie de la matrice discursive dès lors que l'on parle de langue en Acadie. Les « il va de soi », « il est évident », et plus largement l'implicite sont de mise. Dans le corpus, on peut relever 
nombre de mots, expressions, formulations qui induisent l'idée que le discours de l'énonciateur repose sur des évidences, se fonde sur le sens commun. Un seul exemple nous montre le niveau de généralisation d'un propos qui se donne comme une sentence : « le fait de développer le goût des livres ne peut pas faire autrement que d'être positif pour l'alphabétisation dans la province » (AN, 28 octobre 2014). Alors même que le propos fait largement consensus, son énonciateur le présente de façon appuyée dans une logique de l'accumulation, ainsi dans une lettre ouverte une lectrice dénonce les maux du Nouveau-Brunswick:

Le Nouveau-Brunswick a la pire performance économique de toutes les provinces. Nos jeunes partent pour l'Ouest à un rythme alarmant. Les coûts des soins de santé sont immenses, car notre population vieillit et, en moyenne, nous pesons plus, fumons plus et buvons plus que la moyenne nationale. Plus de la moitié des adultes qui habitent ici n'ont pas le niveau d'alphabétisation ou les compétences de travail dont ils ont besoin pour trouver des emplois. (AN, 10 septembre 2014)

À travers ce concert d'accords reviennent certaines images, trois sont récurrentes : celles de la maladie, de la peur et de la lutte. Sans hiérarchie, on note la présence du vocabulaire de la maladie: citoyens infirmes, handicapés, souffrant d'un mal qu'il s'agit de dépister puis de traiter (rappelons l'usage du mot vacciner plus haut), de soigner ; citoyens peu lettrés souffrant aussi de maux physiques comme on a pu le voir plus haut; ceux qui la combattent sont des missionnaires, ceux qui la surmontent de véritables héros comme on le verra plus bas. Relevant du champ lexical de la maladie, on peut citer l'image des « séquelles » qui se « transmettent », du «mal dont on souffre » (dans l'extrait ci-après). La littératie devient alors un ingrédient du bien-être global. Une annonce pour un programme inter-générationnel de bien-être s’énonce ainsi :

Ce programme regorge d'activités amusantes et éducatives en lien avec tous les volets du mieux-être. Pendant 10 semaines, plusieurs intervenants se relayeront pour vous informer et vous guider, et animer une foule d'activités touchant divers aspects du mieux-être (yoga, zumba, activités physiques, alimentation saine, littératie, hygiène, santé mentale et émotionnelle, santé auditive, visuelle et dentaire, et sécurité). [...], les participants pourront s'occuper de divers aspects de leur bien-être (AN, 23 novembre 2018) 
On peut penser aussi à l'activité de levée de fonds communautaire Legs for Litteracy/Courir pour lire dont il est fait promotion de la dernière édition ainsi : «Voilà un événement qui jumelle le mieux-être, le conditionnement physique et la littératie, tous des ingrédients clés afin d’aider les Néo-Brunswickois à améliorer la santé de leur corps et de leur esprit » (AN, 3 novembre 2006) ${ }^{13}$.

On relève aussi le vocabulaire de la peur: une mauvaise littératie est associée à l'échec scolaire puis social et nuisible à l'Acadie tout entière et contre cela, il faut se mobiliser, d'où le thème de la lutte : l'alphabétisation devient une nouvelle terre de mission et la lutte prend les allures d'une véritable guerre sainte. On se rappelle du mot « croisade » cité plus haut ${ }^{14}$. Dans une longue lettre ouverte voici un extrait du portrait de la situation brossé par un acteur communautaire:

«Un peuple illettré est voué à l'assimilation » [... Voilà en quelques mots l'épée de Damoclès qui menace l'Acadie du Nouveau-Brunswick, en particulier.

Le rapport Landal, L'Évaluation intégrale des besoins en formation en alphabétisation au Nouveau-Brunswick, publié en octobre 2002, révélait que parmi les adultes francophones de cette province, 68 \% ne possèdent pas « des capacités suffisantes de lecture, d'écriture et de calcul pour survivre jour après jour dans un monde axé sur l'information écrite » [...]. Ce qui est plus alarmant encore, c'est que, de ce $68 \%$, les trois quarts, environ, sont âgés entre 16 et 25 ans.

Les conséquences de cette situation sont nombreuses et plus tragiques les unes que les autres. Le niveau économique étant directement relié au taux d'analphabétisme du milieu, c'est toute la communauté, toute la province, voire le pays entier, en fin de compte, qui en souffre. Et les séquelles se transmettent généralement d’une génération à l'autre, les capacités en littératie des jeunes adultes n'atteignant même pas, parfois, le niveau de celles de leurs parents, toute proportion gardée, compte tenu des exigences d'une société en mutation. (AN, 18 décembre 2004)

13. Je remercie l'évaluateur qui a suggéré que l'avènement d'un discours médicalisant ainsi que celui qui pose la littératie comme un élément de bien-être global (de l'individu mais aussi de la collectivité) pourrait être mis en lien avec tous les discours de l'art de vivre (« lifestyle») qui semblent parfois remplacer ou du moins doubler des discours plus politisés voire militants. Nous verrons plus loin le traitement très individualisant de la question avec les récits de vie des ex-analphabètes qui se donnent à voir, comme l'a noté l'autre évaluateur, tel des récits exemplaires. Le va et vient entre l'individu et la collectivité est alors constant. Sur le rôle de la responsabilité individuelle en matière de préservation du groupe (dans ses assises linguistiques et nationales) voir Arrighi et Violette, 2013. Dans le cas de figure envisagé dans la contribution de 2013, on voit aussi dépeintes des tranches de vie données comme exemplaires.

14. Notons que cette façon de dépeindre l'analphabétisme, comme un fléau à éradiquer ou une maladie dont les victimes infortunées peuvent être traitées et doivent guérir est dénoncé par certaines instances en charge de l'alphabétisation. Ainsi, un rapport de l'UNESCO souligne les « connotations très négatives de l'analphabétisme [qui] ont stigmatisé ceux qui avaient des compétences faibles en écriture et ont contribué à forger un concept dichotomique plutôt que continu de l'alphabétisme. » (UNESCO, 2006 : 173). 
Indiquons que les thèmes de la maladie, la peur et la lutte sont ceux-là mêmes qui caractérisent de façon plus générale bien des discours sur la qualité de la langue, notamment celui concernant les anglicismes (Bérubé, 2010). Il faut que tout le monde s'engage et lutte, car telle une maladie, l'anglicisme comme l'illettrisme font peur et sont à combattre. Le thème de la lutte est particulièrement développé : il faut se mobiliser, fourbir ses armes et frapper efficacement là où ça fait mal. En 2015, à peine élu, le nouveau gouvernement provincial met sur pied un comité de lutte contre l'analphabétisme nommé selon une rhétorique très en vogue « la Stratégie d'alphabétisation du NouveauBrunswick ». Le premier ministre d'alors, Brian Gallant en a fait l'annonce à l'Assemblée législative et L'Acadie Nouvelle relate la nouvelle:

«Les deux coprésidentes mèneront un processus d'engagement visant à consulter le public et les experts afin de trouver des solutions ingénieuses et transformationnelles pour relever nos défis en matière de littératie », a déclaré Brian Gallant. « Pour soutenir la création d’emplois, accroître la productivité et combattre la pauvreté, nous devons mettre l'accent surtout sur la littératie. » (AN, 11 mars 2015)

Au passage, notons ici la présence d'un thème issu de l'idéo logie néo-libérale voulant que la littératie soit un ingrédient de la réussite économique (des individus mais surtout des communautés). Ainsi, nous retrouvons dans le corpus un discours de l'alphabétisation bureaucratique (selon le mot de Budach, 2003 ${ }^{15}$ ), celui qui promeut l'alphabétisation comme moyen de répondre aux besoins des employeurs et vise l'adaptation de la main-d'œuvre aux nouvelles conditions économiques. Ce faisant, il est question de «niveaux de rendement en littératie » (AN, 21 octobre 2016), de bons ou de mauvais rendements, individus comme gouvernements sont invités à investir en alphabétisation et en littératie, etc.

Enfin, en parallèle à un traitement global et alarmiste de la question, celle-ci est aussi, en même temps, envisagée de façon plus personnelle et positive. Après avoir brossé un tableau désastreux, on travaille à souligner les bons coups, les réussites, on fait une belle part à ces héros du quotidien qui donnent sans compter pour une meilleure littératie en Acadie ou qui se sont dépassés pour sortir de cette condition.

\section{Portrait de l'analphabète}

Présenter la nouvelle par un traitement qui n'accable pas trop la communauté est un mandat implicite du journaliste en milieu minoritaire (Corriveau, 2006 ; Beauchamp et Watine, 2006). Une façon de présenter sous un jour favorable un sujet qui, par ailleurs, fait globalement l'objet d'un traitement négatif est de miser sur le singulier. Le cas personnel, relevant plus ou moins de l'exceptionnel, est

15. Plus qu'une manière d'en parler, ce que Budach (2003) nomme l'alphabétisation bureaucratique, c'est une façon de l'envisager propre au virage néo-libéral. Désormais, les investissements en la matière se doivent d'être rentables, les résultats sont donc évalués, les formateurs professionnalisés, les bénéficiaires rigoureusement sélectionnés selon leur potentiel d'employabilité, etc. 
alors là pour donner le bon exemple. Dans des travaux antérieurs sur les communautés francophones minoritaires en Amérique du Nord, j’avais déjà pu constater avec ma collègue Émilie Urbain qu'une présentation positive de membres de ces communautés voyait le jour dès lorsqu'il s'agissait de promouvoir des activités et initiatives individuelles ou communautaires (Arrighi et Urbain, 2016, 2016/2017). On loue d'autant plus l'agentivité individuelle ou l'efficacité communautaire que les ressources sont maigres, le contexte fortement minoritaire, etc. On propose alors des modèles à suivre sous forme de portrait où le dévouement des uns n'a d'égal que l'héroïsme des autres. Ces panégyriques de bons membres de la communauté sont légion. Ce qui m’a frappé en dépouillant le corpus, c'est le nombre d'articles qui rendent compte de médailles, prix, bourses pour les organismes, institutions, individus qui œuvrent dans le domaine de l'alphabétisation :

Un citoyen de Le Goulet, dans la Péninsule acadienne, fait partie des lauréats régionaux du prix en alphabétisation de l'Association canadienne d'éducation de langue française et de la Fédération canadienne pour l'alphabétisation en français. Donat Roussel recevra une bourse de 100 \$ et un certificat. Un jury composé de trois apprenants et de deux formateurs en alphabétisation a sélectionné les textes en fonction des défis que la personne a réussi à relever. Au total, 86 Canadiens ont participé au prix en alphabétisation. (AN, 26 mai 2004)

Au total, l'Association pour l'alphabétisation du Grand Moncton (Greater Moncton Literacy Advisory Board) a remis six prix à des apprenants adultes, trois dans la catégorie francophone et trois dans la catégorie anglophone. Chacune des auteures en herbe a relaté son parcours, parfois difficile et touchant. (AN, 27 avril 2009)

La représentation des analphabètes que nous offrent les médias est ainsi équivoque. D'une part, on dresse de l'analphabète générique un sombre portrait, d'autre part, on propose de l'analphabète spécifique un portrait plein d'espoir et de lumière. S'alphabétiser, c'est s'améliorer et surtout améliorer la société, comme dit une formatrice citée dans un article : «Il n’y a pas de honte à suivre des cours d'alphabétisation. Des gens éduqués permettent de former une société plus démocratique. L'alphabétisation est là pour répondre aux besoins actuels et futurs du marché du travail. » (AN, 6 mars 2001). Le « choix » de l'alphabétisation est présenté comme un engagement qui suppose un projet de transformation et de réappropriation de sa vie. L'ex-analphabète, celui qui n'a pas eu " honte à suivre des cours d'alphabétisation », est présenté dans le cadre d'une véritable histoire à succès, disant sa résilience et son empowerment. Pour construire un certain pathos, on appuie le côté histoire à succès en rappelant la vie difficile des portraiturés, reprenant à l'échelle individuelle 
les composantes du discours alarmiste mis au jour ci-haut. Nombre d'articles rendent compte de parcours individuels difficiles mais transcendés ${ }^{16}$ :

Mère de trois jeunes enfants, Mélanie Massé a trouvé le courage et la détermination nécessaires pour retourner aux études et obtenir son diplôme d'équivalence d'études secondaires. / Malgré un parcours difficile, elle a su surmonter les obstacles afin de réaliser son rêve, celui d'être acceptée au programme de Techniques d'intervention en délinquance du CCNB. / Mélanie Massé a indiqué vouloir se servir de son expérience de vie afin d’aider les jeunes à retourner sur le droit chemin et à réaliser leurs rêves. (AN, 9 septembre 2015)

"L'alphabétisation a été le commencement de ma vie », avoue Claudette Audet. /Orpheline de père, la jeune fille, huitième d'une famille de neuf enfants, doit à l'époque quitter l'école pour aider sa mère à subvenir aux besoins de la famille. / À l'âge adulte, avec une faible estime d'elle-même, Claudette Audet se replie sur elle-même et ne fréquente les magasins qu'en compagnie d'amis ou de proches et doit demander de l'aide de ses voisins pour les devoirs de ses enfants. / Inscrite à des ateliers d'alphabétisation, la mère de famille reprend peu à peu confiance et est aujourd'hui propriétaire d'un foyer pour soins spéciaux. Mme Audet œuvre également au sein de la Croix-Rouge et recevait, en avril dernier, le prix de l'Apprenante de l'année des mains du ministre de l'Éducation du Nouveau-Brunswick. (AN, 10 septembre 2001)

Claudette Audet, originaire de Val-d'Amour, a remporté le prix de la Francophonie en alphabétisation. Elle a reçu une bourse de 1000 \$ dans le cadre de la Journée internationale de la francophonie. / Mme Audet a mérité le prix pour avoir écrit un texte touchant sur les changements survenus dans sa vie lorsqu'elle a appris à lire, à écrire et à compter. / Le concours s'adressait à des adultes inscrits en alphabétisation. / « Je peux dire que l'alphabétisation a changé ma vie et a probablement sauvé ma vie, a écrit Mme Audet. Je suis plus sociale, je m'isole moins. Du fait que je m'affirme, je suis plus respectée, personne ne peut me dire que je suis une moins que rien, je suis capable de parler et je ne suis plus gênée. » (AN, 21 mars 2002)

Tel un publi-reportage, ce type de portrait, à la faveur de la Journée internationale de l'alphabétisation, se présente sous la forme d'un encadré.

16. Pour avoir fréquenté des organismes en charge de l'alphabétisation, je peux indiquer que l'exercice du portrait fait partie des activités proposées aux bénéficiaires notamment comme moyen de donner sens à sa trajectoire. C'est aussi un « exercice » demandé dans le cadre de certains événements et concours tels ceux mentionnés dans le corps du texte. Le meilleur récit de vie se voyant récompensé. La presse locale publie alors ces portraits comme à l'occasion de la Semaine de la fierté française, elle publie l'autoportrait de bons élèves engagés dans la cause du français (voir Arrighi et Violette, 2013). 


\section{Journée internationale de l'alphabétisation}

\section{Mission accomplie pour Genéva Léger}

Lorsque Genéva Léger de NotreDame-de-Kent a pris sa retraite, une amie lui a conseillé de suivre des cours en alphabétisation. Même si elle savait lire et écrire, Genéva voulait s'améliorer pour réaliser l'un de ses plus grands rêves : travailler sur une collection de photos de famille et raconter son histoire avec des faits vécus. Elle voulait faire ce projet sans faire de fautes d'orthographe.

Mme Léger a travaillé au cours de sa vie comme commis dans un magasin et comme concierge à l'Université de Moncton. Elle a dû faire preuve de persévérance car elle manQuait de confiance lorsou'il était question

d'entreprendre des travaux d'écriture.

* Je riavais pas ma $12^{*}$ année, mais je me suis toujours débrouillée Quand même! l'ai persisté dans mes emplois parce Qu'ils étaient permanents et bien rémunérés.

"Avec la formation en alphabétisation que j'ai reçue, je me vois plutôt comme recherchiste en

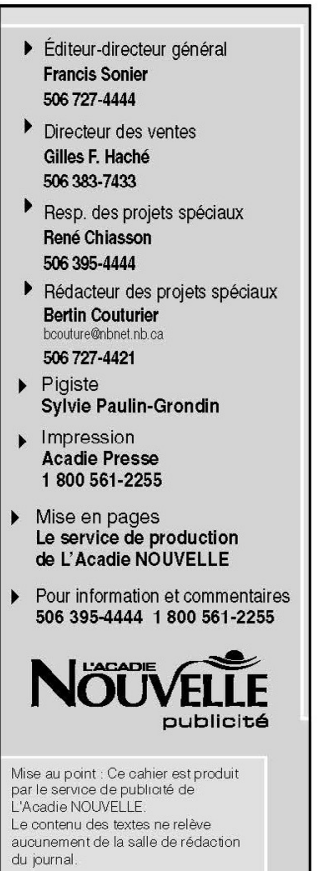

\author{
généalogie. C'est \\ une passion que j'ai \\ découverte lorsque \\ jai suivi mes classes \\ en alphabétisation, 》
}

Mme Léger, Qui a remporté le prix Northup Frye en avril dernier, avai soif de vouloir écrire dans un bon français. Elle est allée dans les classes d'alphabétisation à NotreDame et à Saint-Antoine.

Elle fait partie de la liste des finalistes nationaux dans la catégorie Accomplissement personnel, au
Prix communautaire de l'alphabétisation 2009 de Postes Canada.

Avec la formation en alphabétisation, j'ai plus de confiance en moimême. Par exemple, je suis actuel lement secrétaire du Club de l'âge d'or à Notre-Dame, Queloue chose Que je n'aurais jamais pensé pouvoir faire un jour. Je suis aussi présidente du comité familial du foyer à SainteAntoine. le fais également de la lecture à l'église. Avant, je n'aurais jamais osé lire devant le public. le me surprends à aimer ça et ce sont des qualités que je ne pensais pas Que j'avais. "

Mme Léger encourage fortement toute personne à poursuivre une formation en alphabétisation. Personnellement, un nouveau monde s'est ouvert à moi. Je n'ai

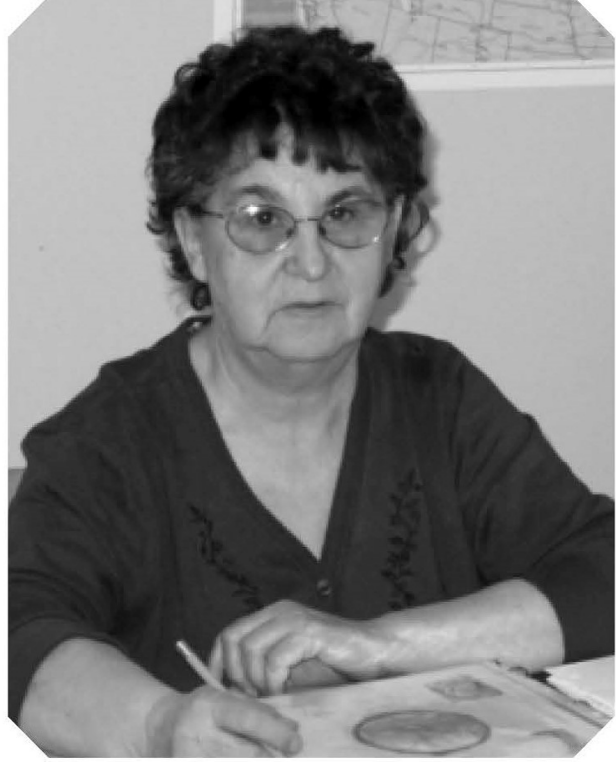

Genéva Léger peut être fière d'elle. Son parcours en alphabétisation lui proarre de belles expériences. pas de mots pour exprimer tout ce oue je ressens. Auparavant, jétais toujours négative et les autres étaient toujours meilleurs
Que moi. Maintenant, je me compte chanceuse : je réalise ou'il y a des gens qui ont vécu des expériences plus pénibles que moi. *
Le conseil d'administration, la directrice générale et les employés de la

Fédération d'alphabétisation du Nouveau-Brunswick profitent de la Journée internationale de l'alphabétisation pour souligner le courage et la

persévérance des apprenants en alphabétisation. Ils tiennent aussi à remercier les personnes qui oeuvrent à l'avancement de l'alphabétisation en français au Nouveau-Brunswick.

Bonne Journée internationale de l'alphabétisation!

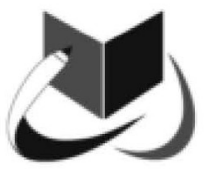

\section{Agir pour garantir l'avenir!}

Fédération d'alphabétisation du Nouveau-Brunswick

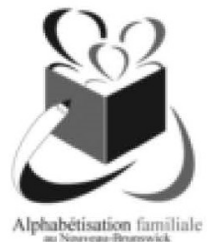
219, rue Main, bureau 8

Bathurst (Nouveau-Brunswick) E2A 1A9

Téléphone : 1866 473-4404 Télécopieur : (506) 548-5564

Courriel : fanb@nbnet.nb.ca $\quad$ Site Web : www.fanb.ca.

IMAge 1 : Portrait de GEnÉva LÉGER PubliÉ dANS L'ACAdIE Nouvelle (8 SEPTEMBRE 2009)

Alphabétisation en milieu de travail Coordonnateur : Reno Michel Haché, M.Éd.

Téléphone : (506) 548-1943
Alphabétisation familiale Coordonnatrice : Réjeanne Cormier Téléphone : (506) 548-1942 
Ce qui ressort c'est un discours qui célèbre la résilience individuelle, met de l'avant le cas particulier pris comme modèle afin de montrer qu'en matière de compétences linguistiques, c'est possible pour tous de s'améliorer avec un peu de bonne volonté. Chacun doit faire sa part pour le plus grand bien de la communauté est un leitmotiv au sein du corpus. On rejoint là le motif de la responsabilité des citoyens en matière de préservation linguistique (Klinkenberg, 2015), discours particulièrement saillant en milieu minoritaire (Arrighi et Violette, 2013). Les compétences linguistiques sont ainsi associées à des attributs personnels (travail, effort, bonne volonté) plutôt qu'à des conditions sociologiques dans la mesure où l'on montre des individus qui ont su dépasser leurs défis de vie. Selon une idéologie forte voulant que l'amélioration des compétences linguistiques soit un processus accessible à tous moyennant un effort personnel (Cameron, 1995 ; Heller, 2007), ces portraits nous montrent comment le perfectionnement individuel est proposé comme clé pour s'en sortir et dépasser des compétences linguistiques jugées déficientes. Pour témoigner de cela, on fait large recours à la parole même de l'analphabète, ce qui est destiné à assoir le crédit du discours proposé.

\section{Pour conclure}

Je voudrais conclure cette contribution sur trois plans différents. En premier lieu, je rappelle que si le traitement médiatique de la question de l'alphabétisation en Acadie (et les idéologies qui soustendent parfois un tel traitement) me parait interrogeable, la pertinence des efforts entrepris par les agents, agentes et bénéficiaires des services dédiés me parait indéniable. S'engager dans un processus d'alphabétisation, c'est s'orienter vers une démarche de sécurisation sociale et langagière. Lise Savoie, chercheuse en travail social, a mené en Acadie un travail auprès de femmes ayant suivi cette voie (2011). Beaucoup de participantes rapportent avoirvécu, lors des sessions d'alphabétisation, des expériences qui ont transformé leur rapport à elles-mêmes et aux autres. Sans avoir nécessairement amélioré leur compétence lettrée, elles disent désormais saisir la possibilité de pouvoir prendre la parole (comme dans les portraits présentés ci-dessus). Cette prise de parole leur permet de développer une nouvelle forme de pouvoir d'agir. Un pouvoir d'agir qui débute avec la prise de conscience du fait qu'elles peuvent redéfinir une image d'elles-mêmes de manière positive. Les participantes racontent qu'elles deviennent des femmes plus autonomes qu'avant et cela se manifeste dans leurs prises de décisions, leur engagement communautaire, la création d'un nouveau sens à leur vie. On est ainsi amené à se demander si l'insécurité ressentie par ces femmes avant leur participation à des sessions d'alphabétisation était le fruit d'un manque de compétences, qui n'a pas toujours été amélioré ou d'un manque de confiance qui lui l'a été. On peut penser notamment que lors de ces sessions, elles ont rencontré un discours alternatif sur l'alphabétisation et la littératie. Ce faisant, non dans l'action en faveur de l'alphabétisation, mais dans les discours qui la promeut, il convient de souligner une certaine prépondérance de propos convenus, des motifs discursifs incontournables et une unanimité d'opinion dont on doit questionner les causes et les enjeux. Ainsi, en second lieu, je voudrais proposer une piste explicative à la prégnance de ce discours posant un déficit linguistique majeur des membres de la communauté acadienne. Au-delà du fait que la déploration du niveau de littératie semble être un sujet largement propagé médiatiquement (Lahire, 1999), pourquoi ce dis- 
cours trouve-t-il en Acadie un si bon écho ? J'ai indiqué dès l'introduction l'ampleur des discours sur les questions linguistiques en Acadie. Plus encore que le statut politique du français comme langue minoritaire, c'est la forme de la langue, sa maitrise par la population, qui est le plus souvent objet de discours et débats. Au Canada français, la question de la langue, de sa qualité, de sa transmission, est éminemment liée à la reproduction et à la pérennité du groupe, à sa survie. C'est une conception à la base du discours national et des revendications de droits des minorités (Heller, 2007), ainsi le discours sur sa qualité ne peut dès lors qu'être très chargé politiquement et émotionnellement. La crainte de disparaitre, où idéologies de la langue et de la nation se mêlent, nous ramène au lien établi entre la langue et le devoir citoyen. Enfin, prompte à accepter qu'elle est en déficit au niveau linguistique, la communauté acadienne semble accepter le diagnostic sur son niveau de littératie telle une donnée irréfutable et non telle une opération d'évaluation, comme un mécanisme au moins en partie subjectif. Il convient donc de se rappeler que les pratiques de littératie sont, comme toutes pratiques et performances socioculturelles, interdépendantes des ordres sociaux et des rapports de pouvoir particuliers où elles apparaissent. À ce sujet, et ce sera mon dernier point, j’ai avancé dès l'introduction que ce discours se comprenait dans une lecture qui, à la suite de Street (1984) notamment, pose l'alphabétisation comme une notion chargée idéologiquement. Reste en partie en suspens le fait de savoir de quelle idéologie relève-t-elle.

Si plusieurs recherches ont mis de l'avant le fait que les attentes en termes de scolarisation et plus particulièrement en termes d'alphabétisation sont modelées par les idéologies linguistiques à l'œuvre au sein d'une société et de ses aspirations en termes politiques, culturels, sociétaux et économiques ${ }^{17}$, il n'en demeure pas moins, qu'en dépit de ces nombreuses recherches, il n'est pas évident de poser quelle grande idéologie reconnue (celle du standard? du monolinguisme ?) sert la notion de littératie.

Étant donné que les discours évaluatifs sur le niveau d'alphabétisation de tel ou tel groupe peuvent servir des intérêts nettement divergents, je propose que ces discours peuvent se mettre au service d'idéologies différentes et même que ces discours permettent de voir poindre des idéologies non encore travaillées par les sociolinguistes.

L’idéologie du standard est assurément concernée dans la mesure où toute valorisation des compétences lettrées est à la fois un ingrédient et un moteur de cette idéologie. Également, comme signalé dans le texte, en faisant de la présence de l'anglais l'une des causes du problème, c'est l'idéologie du monolinguisme qui est servie.

17. Les traditions d'alphabétisation pensées et conduites par l'élite dirigeante sont étroitement liées au contrôle social dans la plupart des sociétés. Pour le passé, on peut mentionner le cas de la mise en place de la scolarisation obligatoire en France où l'apprentissage de la lecture a servi de pierre angulaire à une volonté politique d'unification linguistique (voir Boutet et Fiala, 1978). On peut citer aussi les pratiques coloniales où l'alphabétisation a soutenu les politiques d'acculturation et où le niveau de littératie des peuples colonisés a été instrumentalisé dans un but d'exclusion civique voire civile et de privation du contrôle politique, économique voire parental (voir Woolard et Schieffelin, 1994). 
Quelle (autre) ou quelles (autres) idéologies servent ces discours dans la situation particulière envisagée, et plus largement dans les sociétés occidentales au XXIe siècle ? II n'est pas aisé de répondre définitivement à cette question. En l'état de ma recherche et de ma réflexion, je propose d'y voir à la fois une idéologie (que je nommerai faute de mieux) de la compétence linguistique qui non seulement présuppose que nous disposons des outils pour évaluer ces compétences, que nous pouvons les évaluer sans a priori et que les besoins en la matière seraient déterminés de façon neutre et objective. Cette même idéologie autorise la discrimination sur base linguistique. Par ailleurs, nous avons affaire à une idéologie (que je désignerai toujours faute de mieux) de la bonne langue et des bonnes pratiques linguistiques ${ }^{18}$ qui manifeste une domination culturelle en ce sens que l'alphabétisation dont il est le plus souvent question dans mon corpus renvoie en fait à la maitrise d'une certaine culture lettrée (je rappelle l'absence d'illettré et d'illettrisme comme termes mais non comme référents dans mon corpus). Cette idéologie suppose aussi un niveau optimum ou du moins seuil de compétence en deçà duquel l'individu ne peut être reconnu fonctionnel. Ceci étant si l'idéologie, ou les idéologies, que sert les discours que je viens de présenter est ardue à définir.C'est aussi parce que, pour la ou les déterminer scrupuleusement, il faut tenir compte plus minutieusement que dans la présente contribution, de la figurevoire de la personnalité des discoureurs, de leur occupation, de leur engagement, du moment lors duquel ils prennent la parole, etc. Tous ces paramètres sont encore à creuser dans une exploitation plus en finesse du corpus envisagé. II reste surtout à entreprendre une sociographie auprès des acteurs et actrices de l'alphabétisation en français au Nouveau-Brunswick.

18. On pourrait envisager que cette vision d'un locuteur-scripteur idéal renvoie à l'un des ingrédients de l'idéologie du monolinguisme or, comme le note Ellis (2006), il s'agit d'une idéologie que l'on discute peu notamment en termes de compétences individuelles. En revanche, l'idéologie du bilinguisme qui implique, dans son versant individuel (Heller, 2000), une compétence optimale dans les deux langues comporte cette dimension d'un locuteur scripteur idéal type dont on a montré le caractère illusoire et idéologiquement chargé. 


\section{Références}

\section{Sources primaires}

Brock, Anthony (1971), "Où en est l'alphabétisation dans le monde ? », L'Évangéline, 30 septembre 1971, p. 4.

Dauphin, Damien (2014), « Les élèves encouragés à découvrir la lecture », L’Acadie Nouvelle, 28 janvier 2014, p. 7.

Dupuis, Justin (2008), «L'alphabétisation, un élément important du mieux-être », L’Acadie Nouvelle, 8 septembre 2008, p. 8.

Dyer, Gwynne (2010), « Les objectifs du millénaire », L’Acadie Nouvelle, 22 septembre 2010, p. 19.

Hubert, Jean (1962), « La lutte à l'analphabétisme », L'Évangéline, 16 mai 1962, p. 4.

Lang, Mathieu (2016), "L'alphabétisation doit être au centre de notre projet de société », L’Acadie Nouvelle, 22 septembre 2016, p. 10.

Lyons, Beth (2016), «Services de garde : le soutien essentiel du Gouvernement », L'Acadie Nouvelle, 21 octobre 2016, p. 14.

Marks, Raissa (2014), «En finir avec la pensée conventionnelle », L'Acadie Nouvelle, 10 septembre 2014, p. 14.

Martin, André (2000), «Robert Melanson, le libraire nomade », L’Acadie Nouvelle, 12 mai 2000, p. 6.

Mousseau, Sylvie [SM] (2009), « Brunch littéraire émouvant », L’Acadie Nouvelle, 27 avril 2009, p. 16.

Mousseau, Sylvie (2014), « Le projet Lire et faire lire Acadie célèbre ses cinq ans », L'Acadie Nouvelle, 25 octobre 2014, p. 35.

Roy-Comeau, Mathieu (2015), « Nouveau leadership à l'alphabétisation », L'Acadie Nouvelle, 11 mars 2015, p. 6.

s.a. (1977), « Savent-ils encore lire et écrire? », L'Évangéline, 27 juillet 1977, p. 6.

s.a. (1977), « Un diplômé analphabète », L'Évangéline, 23 septembre 1977, p. 11.

s.a. (1988), «Une association provinciale d'alphabétisation voit le jour », L’Acadie Nouvelle, 3 août 1988, p. 6.

s.a. (2002), « Claudette Audet remporte le prix de la Francophonie en alphabétisation », L’Acadie Nouvelle, 21 mars 2002, p. 5.

s.a. (2004), « Un citoyen de Le Goulet lauréat d'un prix en alphabétisation », L'Acadie Nouvelle, 26 mai 2004, p. 5. 
s.a. (2006), « Dimanche dans le Grand Moncton : courir pour la littératie », L'Acadie Nouvelle, 3 novembre 2006, p. 45.

s.a. (2009), «Trois coopératives du N.-B. mises en évidence», L’Acadie Nouvelle, 15 octobre 2009, p. 15.

s.a. (2009), «Prix Dr Marilyn Trenholme Counssell », L’Acadie Nouvelle, 31 octobre 2009, p. 31.

s.a. (2011), « Clinique pour clients sans médecin de famille », L'Acadie Nouvelle, 25 janvier 2011, p. 23.

s.a. (2011), « La Fédération d’alphabétisation comble un besoin important dans notre société », L'Acadie Nouvelle, 8 septembre 2011, p. 22.

s.a. (2015), « Mission accomplie pour Genéva Léger », L’Acadie Nouvelle, 8 septembre 2015 p. 15.

Shippley, Cyrille (2004), « Un mal social », L’Acadie Nouvelle, 18 décembre 2004, p. 12.

SL (2015), «Deux Néo-Brunswickoises lauréates des Prix de l'Alphabétisation », L’Acadie Nouvelle, 9 septembre 2015, p. 13.

Snow, Claude (Comité des 12) (2015), « Adapter les programmes pour analphabètes à leurs besoins », L'Acadie Nouvelle, 7 août 2015, p. 14.

\section{Sources citées}

Achard, Pierre (1986), « Analyse du discours et sociologie du langage », Langage et société, n 37, p. 5-60.

Arrighi, Laurence et Annette Boudreau (2013), «La construction discursive de l'identité francophone en Acadie ou "comment être francophone à partir des marges" ? ", Minorités linguistiques et société, n³ (La francophonie canadienne en mouvement: continuité ou rupture?, sous la dir. de Pierre Foucher), p. 80-92.

Arrighi, Laurence et Annette Boudreau (2016), « La construction discursive du locuteur francophone : mise en perspective. L'exemple acadien », dans Laurence Arrighi et Annette Boudreau (dir.) Langue et légitimation: la construction discursive du locuteur francophone, Québec, Presses de l’Université Laval, p. 1-18.

Arrighi, Laurence et Émilie Urbain (2016/2017), "Wake up Québec” : du recours aux communautés francophones minoritaires dans le discours visant l'émancipation nationale du Québec », Francophonies d'Amérique, nos 42/43 (Les idéologies linguistiques dans la presse francophone nord-américaine : approches critiques, sous la dir. de Wim Remysen), p. 107-126.

Arrighi, Laurence et Émilie Urbain (2019), «Les “mauvais exemples” de la francophonie nord-américaine : intérêt des acteurs, construction des images, instrumentalisation des groupes », dans Sandrine Hallion et Nicole Rosen (dir.), Les français d'ici : des discours et des usages, Québec, Presses de l'Université Laval, p. 11-32.

Arrighi, Laurence et Isabelle Violette (2013), « De la préservation linguistique et nationale : la qualité de la langue de la jeunesse acadienne, un débat linguistique idéologique », Revue de l'Univer- 
sité de Moncton, vol. 44, nº 2 (Usages, discours et idéologies linguistiques dans la francophonie canadienne : perspectives sociolinguistiques, sous la dir. de Catherine Léger, Matthieu LeBlanc, Laurence Arrighi et Isabelle Violette.), p. 67-101.

Barton, David, Mary Hamilton et Roz Ivanić (dir.) (2000), Situated literacies : reading and writing in context, Londres/New York, Routledge.

Beauchamp, Michel et Thierry Watine (dir.) (2006), Médias et milieux francophones, Québec, Presses de l'Université Laval.

Beaulieu, Gérard (1993), «Les médias en Acadie », dans Jean Daigle (dir.), L'Acadie des maritimes: études thématiques des débuts à nos jours, Moncton, Chaire d'études acadiennes, Université de Moncton, p. 505-542.

Beaulieu, Gérard (dir.) (1997), L'Évangéline 1887-1982 : entre l'élite et le peuple, Moncton, Éditions d'Acadie/Chaire d'études acadiennes.

Bérubé, Julie (2010), Discours et idéologies linguistiques en Acadie du Nouveau-Brunswick: l'exemple de la presse et du milieu scolaire, thèse de doctorat, Université de Moncton.

Boudreau, Annette (2009), «La construction des représentations linguistiques : le cas de l'Acadie », Revue canadienne de linguistique, vol. 54, n³, p. 439-459.

Boudreau, Annette (2011), «La nomination du français en Acadie : parcours et enjeux », dans James de Finney, Hélène Destrempes et Jean Morency (dir.), L'Acadie des origines : mythes et figurations d'un parcours littéraire et historique, Sudbury, Éditions Prise de parole, p. 71-94.

Boudreau, Annette (2012), « Discours, nomination des langues et idéologies linguistiques », dans Davy Bigot, Michael Friesner et Mireille Tremblay (dir.), Les français d'ici : description, représentation et théorisation, Sainte-Foy, Presses de l'Université Laval, p. 89-109.

Boudreau, Annette (2014), « Des voix qui se répondent : analyse discursive et historique des idéologies linguistiques en Acadie : l'exemple de Moncton. », Minorités linguistiques et société, nº 4 (Le français en milieu minoritaire : défis et enjeux. La situation du français en Acadie du NouveauBrunswick, sous la dir. d'Annette Boudreau), p. 175-199.

Boudreau, Annette (2016), Àl'ombre de la langue légitime: l'Acadie dans la francophonie, Paris, Garnier.

Boudreau, Annette et Émilie Urbain (2013), « La presse comme tribune d'un discours d’autorité sur la langue : représentations et idéologies linguistiques dans la presse acadienne, de la fondation du Moniteur Acadien aux Conventions nationales », Francophonies d'Amérique, n 35 (Les journaux des communautés francophones minoritaires en Amérique du Nord, sous la dir. de Dominique Laporte), p. 23-46.

Boutet Josiane et Pierre Fiala (1978), «À propos de ... et il fallut apprendre à écrire à tous les petits français (histoire de la grammaire scolaire) d’A. Chervel », Langage \& société, n³, p. 93-102.

Boutet, Josiane (2001), « La part langagière du travail : bilan et évolution », Langage \& société, n 98, p. 17-42. 
Boutet, Josiane (2007), " La part langagière du travail : théories et analyses », Annuaire de l'EHESS, disponible sur http://journals.openedition.org/annuaire-ehess/18452. [Page consultée le 26 avril 2019.]

Budach, Gabriele (2003), «L'alphabétisation d'adultes en Ontario : la valeur du français entre identité culturelle et ressource économique », dans Monica Heller et Normand Labrie (dir.), Discours et identités : la francité canadienne entre modernité et mondialisation, Louvain-la-Neuve, EME éditions, p. 333-363.

Cameron, Deborah (2007), «Language Endangerment and verbal Hygiene: history, morality and politics », dans Alexandre Duchêne et Monica Heller (dir.), Discourses of endangerment: ideology and interest in the defense of languages, Londres, Continuum, p. 268-285.

Canut, Cécile et Alexandre Duchêne (2011), «Introduction. Instrumentalisations politiques et économiques des langues : le plurilinguisme en question », Langage \& société, nº 136, p. 5-12.

Clerc, Stéphanie et Mireille Rispail (2008), « Former aux langues et aux cultures des autres, une gageure? », Ela : études de linguistique appliquée, vol. 151, n³, p. 277-292.

Collins, James et Richard Blot (2003), Literacy and literacies : text, power and identity, Cambridge, Cambridge University Press.

Corriveau, Claire (2006), «Informer ou défendre la cause : le dilemme de la presse franco-manitobaine », dans Michel Beauchamp et Thierry Watine (dir.), Médias et milieux francophones, Québec, Presses de l'Université Laval, p. 47-64.

Eddie, Marie Hélène (2011), Médias en milieu minoritaires : les attentes et perceptions des publics de l'Acadie du Nouveau-Brunswick envers leur quotidien L'Acadie Nouvelle, thèse de maitrise, Ottawa, Université d'Ottawa.

Eddie, Marie Hélène (2015), «La fermeture du quotidien L'Évangéline en 1982 et la place occupée par le journal dans la mémoire collective acadienne aujourd'hui : entre fatalité, résistance et optimisme », dans Michelle Landry, Martin Pâquet et Anne Gilbert (dir.), Mémoire et mobilisations, Québec, Presses de l'Université Laval, p. 103-124.

Ellis, Elizabeth (2006), «Monolingualism : the unmarked case », Estudios de Sociolingüística, vol. 2, n० 7, p. 173-196.

Espérandieu, Véronique et Jean Vogler (2000), L'illettrisme, Paris, Flammarion.

Fairclough, Norman (1995), Critical Discourse Analysis, Londres, Longman.

Flubacher, Mi-Cha et Alfonso Del Percio (2017), Language, education and neoliberalism : critical studies in sociolinguistics, Londres, Multilingual Matters.

Foucault, Michel (1971), L'ordre du discours, Paris, Gallimard.

Gusfield, Joseph (1981), The culture of public problems : drinking-driving and the symbolic order, Chicago, University of Chicago Press. 
Hambye, Philippe et Anne-Sophie Romainville (2013), "L'appropriation du français : cause ou conséquence d'une intégration réussie », communication présentée à la journée de réflexion L'appropriation du français par les pratiques culturelles, organisée par le Ministère de la Culture, de l'Audiovisuel, de la Santé et de l'Égalité des chances, Molenbeek-Saint-Jean, 20 mars.

Haroche, Claudine, Paul Henry et Michel Pêcheux (1971), "La sémantique et la coupure saussurienne : langue, langage, discours », Langages, n²4, p. 93-106.

Heller, Monica (2000), "Bilingualism and identity in the post-modern world », Estudios de Sociolingüística, vol. 2, nº 1, p. 9-24.

Heller, Monica (2007), "Langue", "communauté" et "identité" : le discours expert et la question du français au Canada », Anthropologie et sociétés, vol. 1, n³1, p. 39-54.

Johnson, Sally et Astrid Esslin (2007), Language in the media : representations, identities, ideologies, Londres/New York, Bloomsbury.

Johnson, Sally et Tomasso Milani (dir.) (2010), Language ideologies and media discourse : texts, practices, politics, Londres, Continuum.

Klinkenberg, Jean-Marie (2015), La langue dans la cité : vivre et penser l'équité culturelle, Bruxelles, Les impressions nouvelles.

Lahire, Bernard (1999), L'invention de l'«illettrisme », Paris, La découverte.

Lahire, Bernard (1992), «Discours sur l'illettrisme et cultures écrites : remarques sociologiques sur un problème social » dans Jean-Marie Besse, Marie-Madeleine De Gaulmyn, Dominique Ginet et Bernard Lahire (dir.), L'«illettrisme» en questions, Lyon, Presses universitaires de Lyon, p. 60-75.

LeBlanc, Mélanie et Annette Boudreau (2016), "Discourses, legitimization, and the construction of Acadianité », Signs and Society, vol. 1, n 4, p. 80-108.

McLaughlin, Mireille (2014), " "I say they don't have education" : industrialisation, mobilité sociale et militantisme linguistique au Canada, 1914-1952 », Langage \& société, n 149, p. 127-142.

Neumann-Holzschuh, Ingrid et Julia Mitko (2018), Grammaire comparée des français d'Acadie et de Louisiane avec un aperçu sur Terre-Neuve, Berlin/Boston, De Gruyter.

OCDE [Organisation de coopération et des développement économiques] (2013), Perspectives de l'OCDE sur les compétences 2013 : premiers résultats de l'évaluation des compétences des adultes, Éditions OCDE, disponible en ligne http://dx.doi.org/10.1787/9789264204096-fr.

Savoie, Lise (2011), Comprendre les trajectoires scolaires de femmes francophones du NouveauBrunswick ayant participé à un processus d'alphabétisation : une perspective féministe, thèse de doctorat, Moncton, Université de Moncton.

Street, Brian (1984), Literacy in theory and practice, Cambridge, Cambridge University Press.

Street, Brian (1995) Social Literacies : critical approaches to literacy development, ethnography and education, Londres, Longman. 
Street, Brian (1996), « Preface », dans Mastin Prinsloo et Mignonne Breir (dir.), The social uses of literacy: theory and practice in contemporary South Africa, Amsterdam, Benjamins, p. 1-10.

Street, Brian (2005), "At last : recent applications of new literacy studies in educational contexts », Research in the teaching of English, vol. 39, n 4, p. 417-423.

Street, Brian (2006), «Autonomous and ideological models of literacy : approaches from new literacy studies », Media Anthropology Network, disponible sur http://www.media-anthropology.net/ street_newliteracy.pdf. [Page consultée le 26 avril 2019.]

UNESCO (2006), «Les défis de l'alphabétisme : un état des lieux », dans Rapport mondial du suivi sur l'éducation pour tous, Paris, Éditions UNESCO, p. 170-199.

Watine, Thierry (1992), «Pratiques journalistiques en milieu acadien : une tradition militante... », dans Fernand Harvey (dir.), Médias francophones hors Québec et identité : analyses, essais et témoignages, Québec, Institut québécois de recherche sur la culture, p. 75-82.

Watine, Thierry (1993), Pratiques journalistiques en milieu minoritaire : la sélection et la mise en valeur des nouvelles en Acadie, thèse de doctorat, Université de Lille III.

Woolard, Kathryn A.et Bambi B. Schieffelin (1994), "Language ideology », Annual Review of Anthropology, vol. 1, n² 23 , p. 55-82. 